\title{
A Modal Logic of Quantification and Substitution
}

\author{
Yde Venema \\ present address: \\ Department of Mathematics and \\ Computer Science \\ Free University \\ De Boelelaan 1081 \\ 1081 HV Amsterdam \\ e-mail: yde@cs.vu.nl
}

\begin{abstract}
.
The aim of this paper is to study the $n$-variable fragment of first order logic from a modal perspective. We define a modal formalism called cylindric mirror modal logic, and show how it is a modal version of first order logic with substitution. In this approach, we can define a semantics for the language which is closely related to algebraic logic, as we find Polyadic Equality Algebras as the modal or complex algebras of our system. The main contribution of the paper is a characterization of the intended 'mirror cubic' frames of the formalisms and, a consequence of the special form of this characterization, a completeness theorem for these intended frames. As a consequence, we find complete finite yet unorthodox derivation systems for the equational theory of finite-dimensional representable polyadic equality algebras.
\end{abstract}

Keywords: algebraic logic, modal logic, logic with finitely many variables, completeness, derivation rules.

1980 Mathematical Subject Classification: 03B20, 03B45, 03C90, 03G15. 


\section{Introduction}

This paper forms part of a program to connect various traditions in logic, viz. first-order logic, modal logic and algebraic logic. In particular, we will show how the $n$-variable fragment of the predicate calculus of $n$-ary relations $(n<\omega)$ can be seen as a modal formalism, which we will call Cylindric Mirror Modal Logic $(C M M L)^{1}$. A distinctive feature of this system is that simultaneous substitutions of variables for variables in first-order formulas are treated as modal diamonds. In this way $C M M L$ is an extension of the formalism $C M L$ (cf. VENEMA [13]) which has diamonds for the existential quantification, and a modal constant for the identity formulas. The connection with algebraic logic lies in the fact that the modal algebras of $C M M L$ are known in the literature as Polyadic Equality Algebras (of finite dimension) (cf. Henkin, Monk \& Tarski [6]).

Note that any modal language can be interpreted in a relational structure of the appropriate signature, i.e. where the structure provides an $n+1$-ary accessibility relation for each $n$-adic operator of the language. Therefore, a consequence of the modal approach towards first-order logic is that it provides us with a wider class of (Kripke) models for first-order logic. Within this more general framework, the standard semantics of first-order logic forms a subclass of frames that will be called mirror cubes here. An interesting aspect of the 'modalization' of first-order logic is that it allows us to play around with this intended semantics; for instance, Andréka and Németi (cf. [2]) have studied an alternative model theory for the predicate calculus where not every $n$-tuple of elements of the domain is available for evaluation; they show that some negative features of first-order logic, like its undecidability, do not apply for this alternative framework.

The main contribution of this note is a finite sound and complete axiomatization of the $C M M L$-formulas that are valid in the mirror cubes. As a consequence of results by Andréka (cf. [1]), a finite axiomatization is not possible, if we confine ourselves to the orthodox modal derivation rules (Modus Ponens, Universal Generalization and Substitution). In fact Andréka and Tuza show [3] that the variety of Representable Polyadic Equality Algebras is not even finitely axiomatizable over the variety of Representable Cylindric Algebras. On the modal side of the picture this means that even if one has an oracle providing all $C M L$-theorems, one still has to add infinitely many axioms to axiomatize the mirror cubes - under the same restriction qua derivation rules. The strategy we adopt here to circumvent these negative results is by considering unorthodox derivation systems. The crucial part of these systems is formed by a so-called non- $\xi$ rule; such rules originate with GABBAY [4], and are discussed in detail in Venema [14]. Our main result concerns the completeness for the class of mirror cubes of a finite unorthodox derivation system. As a corollary of our result, we find a finite derivation system for the variety of Representable Polyadic Equality Algebras of finite dimension. It is interesting to note that this system is a finite extension of a complete derivation system for the Representable Cylindric Algebras. One might conclude that the heart of the nonfinite axiomatizability problem of RPEA does not lie in its complexity with respect to RCA, but rather in the inadequacy of a purely equational approach to axiomatizations in algebraic logic.

Therefore, the question becomes relevant what the exact algebraic counterpart is of non- $\xi$ rules. We will come back to this matter in section 4 , where we will also discuss briefly some

\footnotetext{
${ }^{1}$ One can approach the unrestricted predicate calculus from the same modal perspective. As there are many (mainly technical) problems involved in doing so, we have confine ourselves to a fragment of first-order logic here.
} 
generalizations to our results that were obtained recently by Sz. Mikulás.

This paper is organized as follows: in the following section we go into detail as to how the $n$-variable fragment of the predicate calculus of $n$-ary relations $(n<\omega)$ can be 'modalized' into the formalism $C M M L$. In section 3 we introduce a relational (Kripke) semantics for our language, and we prove our main completeness result. Section 4 contains all the material on the algebraic connection: in particular, we define the finite derivation system for the equational theory of the class RPEA ${ }_{n}$ of Representable Polyadic Equality Algebras.

\section{Modalizing first-order logic}

In order to explain how the $n$-variable fragment of the predicate calculus of $n$-ary relations can be treated as a modal formalism, let us start with an intuitive exposition, and defer precise definitions to the end of this section; until then, the reader can think of a version of first-order logic, where only the first $n$ variables $\left\{v_{0}, \ldots, v_{n-1}\right\}$ are available, with the standard semantics of first-order logic. Consider the basic declarative statement in first-order logic concerning the truth of a formula in a model under an assignment $u$ :

$$
\mathfrak{M} \models \phi[u] .
$$

The basic observation underlying our approach is that we can read (1) from an abstract modal perspective as: "the formula $\phi$ is true in $\mathfrak{M}$ at the possible world $u$ ". Note that as we have only $n$ variables at our disposal, we can identify assignments with maps: $n(=\{0, \ldots, n-1\}) \mapsto U$, or equivalently, with $n$-tuples over the domain $U$ of the structure - we will denote the set of such $n$-tuples with ${ }^{n} U$. Thus we are in a setting of multi-dimensional modal logic where the universe of a modal model is of the form ${ }^{n} U$ for some base set $U$. Now the truth definition of the quantifiers reads as follows:

$$
\mathfrak{M} \models \exists v_{i} \phi[u] \quad \Longleftrightarrow \quad \text { there is an assignment } u^{\prime} \text { with } u \equiv_{i} u^{\prime} \text { and } \mathfrak{M} \models \phi\left[u^{\prime}\right]
$$

where $\equiv_{i}$ is given by

$$
u \equiv_{i} u^{\prime} \Longleftrightarrow \text { for all } j \neq i, u_{j}=u_{j}^{\prime} .
$$

In other words: the existential quantification behaves like a modal diamond, having $\equiv_{i}$ as its accessibility relation.

As the semantics of the boolean connectives in the predicate calculus is the same as in modal logic, this shows that the inductive clauses in the truth definition of first-order logic fit neatly in a modal approach. So let us now concentrate on the atomic formulas. To start with, we observe that identity formulas do not cause any problem: a formula $v_{i}=v_{j}$, with truth definition

$$
\mathfrak{M} \models v_{i}=v_{j}[u] \Longleftrightarrow u_{i}=u_{j}
$$

can be seen as a modal constant.

The case of the other atomic formulas is more involved, however; as we have confined ourselves to the calculus of $n$-adic relations, an atomic predicate formula is of the form $P_{i} v_{\nu(0)} \ldots v_{\nu(n-1)}$, where $\nu$ is a map: $n \mapsto n$. In the model theory of first-order logic the predicate $P_{i}$ will be interpreted as a subset of ${ }^{n} U$; precisely how the propositional variables are treated in modal logic by a valuation. So we will identify the set of propositional variables of the modal formalism with the set of predicate symbols of our first-order language. However, this implies that there cannot be a one-to-one correspondence between atomic first-order 
formulas and atomic modal ones. It follows from our wish to give a modal reading for the atomic case of (1), that only the formula $P_{i} v_{0} \ldots v_{n-1}$ will correspond to the modal atom $p_{i}$. For the cases where $\nu$ is not the identity function, we have to find a different solution.

Atomic formulas with a multiple occurrence of a variable can be rewritten as formulas with only 'unproblematic' atomic subformulas, for instance

$$
\begin{aligned}
P v_{1} v_{0} v_{0} & \Longleftrightarrow \exists v_{2}\left(v_{2}=v_{0} \wedge P v_{1} v_{2} v_{2}\right) \\
& \Longleftrightarrow \exists v_{2}\left(v_{2}=v_{0} \wedge \exists v_{0}\left(v_{0}=v_{1} \wedge P v_{0} v_{2} v_{2}\right)\right) \\
& \Longleftrightarrow \exists v_{2}\left(v_{2}=v_{0} \wedge \exists v_{0}\left(v_{0}=v_{1} \wedge \exists v_{1}\left(v_{1}=v_{2} \wedge P v_{0} v_{1} v_{2}\right)\right)\right)
\end{aligned}
$$

This leaves the case what to do with atoms of the form $P_{i} v_{\sigma(0)} \ldots v_{\sigma(n-1)}$, where $\sigma$ is a permutation of $n$, or in other words, atomic formulas where variables have been substituted simultaneously. The previous trick does not work here: for instance, to write a formula like

$$
\exists v_{3} \exists v_{4}\left(v_{3}=v_{0} \wedge v_{4}=v_{1} \wedge \exists v_{0} \exists v_{1}\left(v_{0}=v_{4} \wedge v_{1}=v_{3} \wedge P v_{0} v_{1} v_{2}\right)\right),
$$

which is equivalent to $P v_{1} v_{0} v_{2}$, one needs extra variables as buffers.

One might consider a solution where a predicate $P$ is translated into various modal propositional variables $p_{\sigma}$, one for every permutation $\sigma$ of $n$, but this is not very elegant. One might also forget about simultaneous substitutions and confine oneself to a fragment of $n$-variable logic. In VenEma [13] this option is worked out, leading to a modal formalism called Cylindric Modal Logic.

Here we will investigate a third possibility, which is to take substitution seriously, so to speak, by adding special 'substitution operators' to the language. The crucial observation is that for any permutation $\sigma$, we have that

$$
\mathfrak{M} \models P_{i} v_{\sigma(0)} \ldots v_{\sigma(n-1)}[u] \Longleftrightarrow \mathfrak{M} \models P_{i} v_{0} \ldots v_{n-1}[\sigma \circ u]
$$

where $\sigma \circ u$ is the composition ${ }^{2}$ of $\sigma$ and $u$ (recall that $u$ is a map: $n \mapsto U$ ). So, if we define the relation $\bowtie_{\sigma} \subseteq{ }^{n} U \times{ }^{n} U$ by

$$
u \bowtie_{\sigma} t \Longleftrightarrow t=\sigma \circ u
$$

we have rephrased (2) in terms of an accessibility relation (in fact, a function):

$$
\mathfrak{M} \models P_{i} v_{\sigma(0)} \ldots v_{\sigma(n-1)}[u] \Longleftrightarrow \text { there is a } t \text { with } u \bowtie_{\sigma} t \text { and } \mathfrak{M} \models P_{i} v_{0} \ldots v_{n-1}[t]
$$

So if we add an operator $\otimes_{\sigma}$ to the modal language for every permutation $\sigma$, with $\bowtie_{\sigma}$ as its intended accessibility relation, we have found the desired modal equivalent for a formula $P_{i} v_{\sigma(0)} \ldots v_{\sigma(n-1)}$ in the form $\otimes_{\sigma} P_{i}$.

Our last observation before we give the formal definitions of our systems is that we may use the fact that we are in a finite-variable fragment of first-order logic to simplify the language a bit. For, recall that every permutation of a finite set is a product of transpositions, i.e. permutations swapping two elements and leaving every other element in its place. As we may infer from (2) that

$$
\mathfrak{M} \models P_{i} v_{\tau \circ \sigma(0)} \ldots v_{\tau \circ \sigma(n-1)}[u] \Longleftrightarrow \mathfrak{M} \models P_{i} v_{0} \ldots v_{n-1}[\tau \circ(\sigma \circ u)]
$$

\footnotetext{
${ }^{2}$ In our notation, the order of composing the two functions should be read as in the composition of two relations, i.e., $\sigma \circ u(i)=u(\sigma(i))$.
} 
we only need modal operators $\otimes_{\tau}$ for transpositions $\tau$ : if $\sigma=\tau_{0} \circ \ldots \tau_{k}$, we may consider $\otimes_{\sigma}$ as an abbreviated operator:

$$
\otimes_{\sigma} \phi:=\otimes_{\tau_{0}} \ldots \otimes_{\tau_{n-1}} \phi
$$

So, to develop some notation concerning transpositions and their associated accessibility relations: define the transposition $[i, j]^{n}: n \mapsto n$ by

$$
[i, j]^{n}(k)= \begin{cases}j & \text { if } k=i \\ i & \text { if } k=j \\ k & \text { otherwise }\end{cases}
$$

(If no confusion can arise, we will drop the superscript $n$.) The accessibility relation associated with transpositions has a very simple form (assume $i<j$ ):

$$
u \bowtie_{i j} v \Longleftrightarrow v=\left(u_{0}, \ldots, u_{i-1}, u_{j}, u_{i+1}, \ldots, u_{j-1}, u_{i}, u_{j+1}, \ldots, u_{n_{1}}\right) .
$$

Now we are ready to give formal definitions:

Definition 2.1 Let $n$ be an arbitrary but fixed natural number. The alphabet of $L_{n}$ and of $L_{n}^{r}$ consists of a set of variables $\left\{v_{i} \mid i<n\right\}$, it has got a countable set $Q$ of $n$-adic relation symbols $\left(P_{0}, P_{1}, \ldots\right)$, identity $(=)$, the Boolean connectives $\neg, \vee$ and the quantifiers $\exists v_{i}$. Formulas of $L_{n}$ and $L_{n}^{r}$ are defined as usual in first-order logic, with the restriction that the atomic formulas of $L_{n}^{r}$ are of the form $v_{i}=v_{j}$ or $P_{l}\left(v_{0} v_{1} \ldots v_{n}\right)$; for $L_{n}$, we also allow atomic formulas of the form $P_{l}\left(v_{\sigma 0} v_{\sigma 1} \ldots v_{\sigma n}\right)$, where $\sigma$ is a permutation on $n$.

A first-order structure for $L_{n}^{(r)}$ is a pair $\mathfrak{M}=(U, V)$ such that $U$ is a set called the domain of the structure and $V$ is an interpretation function mapping every $P_{l}$ to a subset of ${ }^{n} U$. Truth of a formula in a model is defined as usual: let $u$ be in ${ }^{n} U$, then

$$
\begin{array}{lll}
\mathfrak{M} \models v_{i}=v_{j}[u] & \text { if } u_{i}=u_{j}, \\
\mathfrak{M} \models P_{l}\left(v_{0} v_{1} \ldots v_{n}\right)[u] ; & \text { if } u \in V\left(P_{l}\right), \\
\mathfrak{M} \models P_{l}\left(v_{\sigma 0} v_{\sigma 1} \ldots v_{\sigma n}\right)[u] & \text { if }\left(u_{\sigma(o)}, \ldots, u_{\sigma(n-1)}\right) \in V\left(P_{l}\right), \\
\mathfrak{M} \models \exists v_{i} \phi[u] & \text { if there is a v with } u \equiv_{i} v \text { and } \mathfrak{M} \models \phi[v], \\
\text { etc. } & &
\end{array}
$$

An $L_{n}^{(r)}$-formula $\phi$ is valid in $\mathfrak{M}$ (notation: $\mathfrak{M} \models \phi$ ) if $\mathfrak{M} \models \phi[u]$ for all $u \in{ }^{n} U$, first-order valid (notation: $\models_{\text {fo }} \phi$ ) if it is valid in every first-order structure of $L_{n}^{(r)}$.

The modal versions of $L_{n}^{r}$ and $L_{n}$, on which we will concentrate from now on, are given in the following definition:

Definition 2.2 Let $n$ be an arbitrary but fixed natural number. $C M M L_{n}$ is the modal similarity type having constants $\delta_{i j}$ and unary operators $\diamond_{i}, \otimes_{i j}$ (for all $i, j<n$ ). For a set of propositional variables $Q$, the language of $n$-dimensional cylindric modal formulas in $Q$, or shortly, $C M M L_{n}$-formulas (in $Q$ ), is built up as usual: the atomic formulas are the (modal or boolean) constants and the propositional variables, and a formula is either atomic or of the form $\neg \phi, \phi \vee \psi, \diamond_{i} \phi$ or $\otimes_{i j} \phi$, where $\phi, \psi$ are formulas. We abbreviate $\square_{i} \phi \equiv \neg \diamond_{i} \neg \phi . C M L_{n}$ is the fragment of $C M M L_{n}$-formulas in which no mirror operator $\otimes_{i j}$ occurs. 
$C M L$ and $C M M L$ are interpreted in first-order structures in the obvious way; for instance we have

$$
\begin{aligned}
& \mathfrak{M}, u \Vdash \delta_{i j} \quad \Longleftrightarrow u_{i}=u_{j} \\
& \mathfrak{M}, u \Vdash \otimes_{i j} \phi \quad \Longleftrightarrow \quad \text { there is a } v \text { with } u \bowtie_{i j} v \text { and } \mathfrak{M}, v \Vdash \phi \\
& \mathfrak{M}, u \Vdash \diamond_{i} \phi \quad \Longleftrightarrow \quad \text { there is a v with } u \equiv_{i} v \text { and } \mathfrak{M}, v \Vdash \phi
\end{aligned}
$$

A $C M M L_{n}$-formula $\phi$ is valid, notation: $\mathrm{MC}_{n} \models \phi$, if it valid in all first-order structures ${ }^{3}$.

The modal disguise of $L_{n}^{r}$ in $C M M L_{n}$ is so thin, that we feel free to give the details below without further comments resp. proof:

Definition 2.3 Let $\mu$ be the following translation from $L_{n}$ to $C M M L_{n}$ :

$$
\begin{array}{ll}
\mu\left(P v_{0} \ldots v_{n-1}\right) & =p \\
\mu\left(P v_{\sigma(0)} \ldots v_{\sigma(n-1)}\right) & =\otimes_{r_{1} s_{1}} \ldots \otimes_{r_{k} s_{k}} p \\
\mu\left(v_{i}=v_{j}\right) & =\delta_{i j} \\
\mu(\neg \phi) & =\neg \mu(\phi) \\
\mu(\phi \vee \psi) & =\mu(\phi) \vee \mu(\psi) \\
\mu\left(\exists v_{i} \phi\right) & =\diamond_{i} \mu(\phi)
\end{array}
$$

where we assume that we have a canonical definition of numbers $r_{1}, \ldots, r_{k}$ and $s_{1}, \ldots, s_{k}$ such that $\sigma=\left[r_{1} s_{1}\right] \circ \ldots \circ\left[r_{k} s_{k}\right]$.

Proposition 2.4 Let $\phi$ be a formula in $L_{n}^{r}$, then

$$
\models_{\text {fo }} \phi \Longleftrightarrow \mathrm{MC}_{n} \models \mu(\phi) \text {. }
$$

Note that the modal language is in fact the stronger formalism: where in first-order logic, the simultaneous substitution of two variables for each other can only be defined by induction, in $C M M L$ it is a primitive operator.

\section{Completeness}

In this section we discuss the modal semantics and axiomatics of $C M M L_{n}$ (and hence, of $L_{n}$ ). To start with the semantics, recall that in our intended $n$-dimensional structures for $C M M L_{n}$, the accessibility relations $I d_{i j}, \equiv_{i j}$ and $\bowtie_{i j}$ for $\delta_{i j}, \diamond_{i j}$ and $\otimes_{i j}$ are given as

$$
\begin{aligned}
& u \in I d_{i j} \Longleftrightarrow u_{i}=u_{j} \\
& u \equiv_{i} v \Longleftrightarrow \text { for all } j \neq i: u_{j}=v_{j} \\
& u \aleph_{i j} v \Longleftrightarrow u_{i}=v_{j}, u_{j}=v_{i} \& \text { for all } k \neq i, j: u_{k}=v_{k} .
\end{aligned}
$$

In the relational semantics for $C M M L_{n}$ we abstract away from this background and define models where the universe is an arbitrary set and the accessibility relations are arbitrary relations, of the appropriate arity. The key questions then are how to distinguish the intended structures within this more general semantics, and how to axiomatize their theory. For the $C M L$-fragment of the language, these questions have been discussed in detail in VENEMA [13]. The main results of that paper will be used here, as we will take the characterization and axiomatization results of the $C M L$-cubes as a starting point.

\footnotetext{
${ }^{3}$ This definition is slightly ambiguous. For, let $m, n$ be two natural numbers with $m<n$, and $\phi$ a $C M M L_{m^{-}}$ formula. Then by definition $\phi$ is also a $C M M L_{n}$-formula. So, when checking the validity of $\phi$, in principle it might make a difference whether one considers assignments in ${ }^{m} U$ or in ${ }^{n} U$. A fairly straightforward proof showing this not to be the case, justifies our definition.
} 
Definition 3.1 A $C M M L_{n}$-frame or simply a frame, is a tuple $\left(W, T_{i}, E_{i j}, M_{i j}\right)_{i, j<n}$, where $E_{i j}$ is a subset of the universe $W$, and $T_{i}$ and $M_{i j}$ are binary relations over $W$. A $\left(C M M L_{n^{-}}\right.$ )model is a pair $\mathfrak{M}=(\mathfrak{F}, V)$ with $\mathfrak{F}$ a frame and $V$ a valuation, i.e. a map assigning subsets of $W$ to propositional variables. The cylindric reduct of a frame $\mathfrak{F}=\left(W, T_{i}, E_{i j}, M_{i j}\right)_{i, j<n}$, notation $\mathfrak{F}_{\text {cyl }}$, is the tuple obtained from $\mathfrak{F}$ by suppressing all the mirror relations $M_{i j}$, i.e. $\mathfrak{F}_{\text {cyl }}=$ $\left(W, T_{i}, E_{i j}\right)_{i, j<n}$. A CMML -frame is called a mirror cube if it is isomorphic to a frame of the form $\left({ }^{n} U, \equiv_{i}, I d_{i j}, \aleph_{i j}\right)_{i, j<n}$, where $\equiv_{i}, I d_{i j}$ and $\aleph_{i j}$ are as defined above. Cylindric reducts of mirror cubes are called cubes. The classes of n-dimensional mirror cubes and cubes are denoted as $\mathrm{MC}_{n}$ resp. $\mathrm{C}_{n}$.

Truth of a formula at a world is defined as usual in modal logic:

$\begin{array}{ll}\mathfrak{M}, w \Vdash p & \Longleftrightarrow w \in V(p), \\ \mathfrak{M}, w \Vdash \delta_{i j} & \Longleftrightarrow w \in E_{i j}, \\ \mathfrak{M}, w \Vdash \neg \phi & \Longleftrightarrow \mathfrak{M}, w \Vdash \phi, \\ \mathfrak{M}, w \Vdash \phi \wedge \psi & \Longleftrightarrow \mathfrak{M}, w \Vdash \phi \& \mathfrak{M}, w \Vdash \psi, \\ \mathfrak{M}, w \Vdash \diamond_{i} \phi & \Longleftrightarrow \text { there is a vith } T_{i} w v \& \mathfrak{M}, v \Vdash \phi, \\ \mathfrak{M}, w \Vdash \otimes_{i j} \phi & \Longleftrightarrow \text { there is a vith } M_{i j} w v \& \mathfrak{M}, v \Vdash \phi .\end{array}$

Validity of a formula/set of formulas in a model/frame or class of models/frames, is defined as usual; for instance, $\mathrm{MC}_{n} \models \phi$ denotes validity of $\phi$ in the class of miror cubes ${ }^{4}$.

This definition provides us with an alternative, more general semantics for the $n$-variable fragment of the predicate calculus as well. This more abstract perspective on first-order logic allows one to play more freely with the intended structures for $L_{n}^{(r)}$. For instance, Andréka and Németi [2] argue that $L_{n}^{r}$ behaves in a much nicer way if we allow relativized (mirror) cubes as models, i.e. not every tuple $\left(u_{0}, \ldots, u_{n-1}\right)$ in ${ }^{n} U$ is available as a possible world of the model. For instance, they show that for this relativized-cube semantics both a decidability and a finite axiomatizability result can be obtained.

Now, to start with a characterization of the mirror cubes, consider the following formulas:

Definition 3.2 We define the following $C M M L_{n}$-formulas, and their counterparts in the associated first-order language for talking about frames:

$$
\begin{aligned}
& \left(M 1_{i j}\right) \quad \otimes_{i j} p \leftrightarrow \neg \otimes_{i j} \neg p \\
& \left(M 2_{i j}\right) \quad p \rightarrow \neg \otimes_{i j} \neg \otimes_{i j} p \\
& \left(M 3_{i j}\right) \otimes_{i j} p \rightarrow\left[\diamond_{i}\left(\delta_{i j} \wedge \diamond_{j} p\right) \wedge \diamond_{j}\left(\delta_{i j} \wedge \diamond_{i} p\right)\right] \\
& \left(M 1_{i j}^{s}\right) \quad \forall u \exists ! v M_{i j} u v \\
& \left(M 2_{i j}^{s}\right) \quad \forall u v\left(M_{i j} u v \rightarrow M_{i j} v u\right) \\
& \left(M 3_{i j}^{s}\right) \quad \forall u v\left(M_{i j} u v \rightarrow\left[\exists w\left(T_{i} u w \wedge E_{i j} w \wedge T_{j} w v\right) \wedge \exists w\left(T_{j} u w \wedge E_{i j} w \wedge T_{i} w v\right)\right]\right.
\end{aligned}
$$

We set $M 1=\bigwedge_{i, j<n} M 1_{i j}$, etc.

Proposition 3.3 Let $\mathfrak{F}=\left(W, T_{i}, E_{i j}, M_{i j}\right)_{i, j<n}$ be a frame. Then

(i) $\mathfrak{F} \models M k_{i j} \Longleftrightarrow \mathfrak{F}$ satisfies $M k_{i j}^{s}$, for $k=1,2$ or 3 , and $i, j<n$.

(ii) $\mathfrak{F}$ is a mirror cube iff $\mathfrak{F}_{\text {cyl }}$ is a cube and $\mathfrak{F}$ satisfies $M 1, M 2$ and $M 3$.

\footnotetext{
${ }^{4}$ It may seem that our notation has become even more ambiguous (cf. footnote 3). For, now we use ' $M C_{n} \models \phi$ ' to denote both validity of $\phi$ in first-order structures (Definition 2.2) and validity of $\phi$ in a class of modal frames, viz. the mirror cubes. It is easy to see that this ambiguity is only apparent, due to the technical fact that the domain $U$ of a first-order structure is not identical to the universe ${ }^{n} U$ of the corresponding $n$-dimensional mirror cube.
} 


\section{Proof.}

(i) This is immediate by the fact that all $M$-formulas are standard Sahlqvist forms, cf. SAHLQVIST [12], or VENEMA [14].

(ii) We only show the direction from right to left. Let $\mathfrak{F}$ be a frame such that $\mathfrak{F}_{\text {cyl }}$ is a cube, and such that all $M$-formulas are valid in $\mathfrak{F}$. We may assume that $\mathfrak{F}$ is of the form $\left({ }^{n} U, \equiv_{i}, I d_{i j}, M_{i j}\right)_{i, j<n}$. From (i) it follows that $\mathfrak{F}$ satisfies $M 1^{s}$ and $M 3^{s}$. As $M_{i j}$ is functional, we may write $m_{i j}(u)$ for the unique $v$ such that $M_{i j} u v$. To show that $\mathfrak{F}$ is a mirror cube, we have to prove that for arbitrary $u, v$ in ${ }^{n} U$ :

$$
v=m_{i j}(u) \Longleftrightarrow u \bowtie_{i j} v .
$$

From left to right: Without loss of generality we may assume that $i=0, j=1$; let $v=m_{01}(u)$. By the validity of $M 3^{s}$ in $\mathfrak{F}$, and the fact that $\mathfrak{F}_{c y l}$ is a cube, we find tuples $w, w^{\prime}$ such that $u \equiv_{0} w, w_{0}=w_{1} \& w \equiv_{1} v$ and $u \equiv_{1} w^{\prime}, w_{0}^{\prime}=w_{1}^{\prime} \& w^{\prime} \equiv_{0} v$. From $u \equiv_{0} w$ and $w_{0}=w_{1}$ it follows that $w$ is of the form $w=\left(u_{1}, u_{1}, u_{2}, \ldots, u_{n-1}\right)$, so the fact that $w \equiv_{1} v$ gives $u_{1}=v_{0}$ and $u_{k}=v_{k}$ for $k>1$. Analogous reasoning with $w^{\prime}$ yields that $u_{0}=v_{1}$, so indeed we have found $u \bowtie_{i j} v$.

From right to left: In a cube, the condition on the righthandside of $(*)$ determines a unique $v$ for a given $u$. So $m_{i j}(u)$ (which exists by the fact that $\mathfrak{F}$ satisfies $M 1^{s}$ ), must be identical to $v$, as both $m_{i j}(u)$ and $v$ satisfy this condition.

Proposition 3.3(ii) forms the crucial observation in our completeness proof below. Before we proceed to define the derivation system and prove its completeness, let us briefly discuss two essential aspects of it: (i) the $D$-operator, and (ii) the non- $\xi$ rules.

To start with (i): the $D$-operator is a diamond with a rather special accessibility relation, viz. the inequality relation $(\neq)$ :

$$
\mathfrak{M}, w \Vdash D \phi \Longleftrightarrow \text { there is a different } v \text { (i.e. } w \neq v \text { ) with } \mathfrak{M}, v \Vdash \phi \text {. }
$$

The $D$-operator enlarges the expressive power of modal languages; for instance, it enables one to define irreflexivity of the accessibility relation, viz. by the formula $\diamond p \rightarrow D p$. For more general information about the $D$-operator, we refer to DE RIJKE [11].

In the present context, we are so fortunate to have the $D$-operator as a defined operator, at least over the class of (mirror) cubes: set

$$
D_{n} \phi=\bigvee_{j \neq i} \diamond_{j}\left(\delta_{i j} \wedge \diamond_{i}\left(\neg \delta_{i j} \wedge \diamond_{0} \ldots \diamond_{i-1} \diamond_{i+1} \ldots \diamond_{n-1} \phi\right)\right)
$$

In Venema [13] we show that for a cube model $\mathfrak{M}$, indeed we have that $D_{n} \phi$ is true at a world iff there is a different world where $\phi$ holds. (The key observation here is that two $n$-tuples are different iff they differ in at least one coordinate.)

Note that in fact, we can associate an accessibility relation with $D_{n}$ in arbitrary frames (i.e. define a relation $R_{D_{n}}$ in terms of the relations $T_{i}$ and $E_{i j}$ ) such that in a model $\mathfrak{M}$ based on $\mathfrak{F}$, we have $\mathfrak{M}, w \Vdash D_{n} \phi$ iff there is a $v$ with $R_{D_{n}} w v$ and $\mathfrak{M}, v \Vdash \phi$. One of the main results of VENEM A [13] states that a frame $\mathfrak{F}$ is isomorphic to a cube iff $\mathfrak{F}$ satisfies some straightforward properties expressible by modal formulas, plus the fact that $R_{D_{n}}$ is the inequality relation.

Turning to axiomatics, this is the spot where the unorthodox derivation rules (mentioned in the introduction) will come in. To give some intuitions, let us consider the problem how to axiomatize irreflexivity in a standard modal context with one diamond $\diamond$. It is well-known 
that there is no axiom for irreflexivity in the way that $\diamond \diamond p \rightarrow \diamond p$ axiomatizes transitivity, so we have to take resort to different means. Consider a formula $\phi$ which is satisfiable in an irreflexive frame $\mathfrak{F}$, i.e. there is a valuation $V$ and a world $w$ such that $\mathfrak{F}, V, w \Vdash \phi$. As we have $\neg R w w$, we find that $\mathfrak{F}, V^{\prime}, w \Vdash p \wedge \neg \diamond p$ under any valuation $V^{\prime}$ with $V^{\prime}(p)=\{w\}$. Now if we take a propositional variable $p$ which does not occur in $\phi$, it will be clear that we can combine $V$ and $V^{\prime}$ into a valuation $V^{\prime \prime}$ for which $\mathfrak{F}, V^{\prime \prime}, w \Vdash \phi \wedge(p \wedge \neg \diamond p)$. In other words: $\phi \wedge(p \wedge \neg \diamond p)$ is satisfiable. Turning from satisfiability to validity, we have shown that if $(p \wedge \neg \diamond p) \rightarrow \neg \phi$ is valid, then so is $\neg \phi$. Or equivalently, the derivation rule " $\vdash(p \wedge \neg \diamond p) \rightarrow \psi \Rightarrow \vdash \psi$, provided that $p$ does not occur in $\psi$ ", is sound. In VenEma [14], it is proved that under certain conditions, these rules will also give complete calculi.

Now we are ready to define an axiom system for the mirror cubes, and prove its completeness:

Definition 3.4 $A_{n}^{+}$is the derivation system with the following axioms:

$$
\begin{array}{ll}
\left(C M 1_{i}\right) & p \rightarrow \diamond_{i} p \\
\left(C M 2_{i}\right) & p \rightarrow \square_{i} \diamond_{i} p \\
\left(C M 3_{i}\right) & \diamond_{i} \diamond_{i} p \rightarrow \diamond_{i} p \\
\left(C M 4_{i j}\right) & \diamond_{i} \diamond_{j} p \rightarrow \diamond_{j} \diamond_{i} p \\
\left(C M 5_{i}\right) & \delta_{i i} \\
\left(C M 6_{i j}\right) & \left.\diamond_{i}\left(\delta_{i j} \wedge p\right) \rightarrow \square_{i}\left(\delta_{i j} \rightarrow p\right)\right) \\
\left(C M 7_{i j k}\right) & \delta_{i j} \rightarrow \diamond_{k}\left(\delta_{i k} \wedge \delta_{k j}\right) \\
\left(C M 8_{i j}\right) & \left(\delta_{i j} \wedge \diamond_{i}\left(\neg p \wedge \diamond_{j} p\right)\right) \rightarrow \diamond_{j}\left(\neg \delta_{i j} \wedge \diamond_{i} p\right)
\end{array}
$$

Its orthodox derivation rules are Modus Ponens, Universal Generalization and Substitution:

$(M P) \quad \phi, \phi \rightarrow \psi / \psi$

$(U G) \quad \phi / \square \phi \quad$ for $\square \in\left\{\square_{i} \mid i<n\right\}$

$(S U B) \phi / \sigma \phi, \quad$ for any substitution $\sigma$ of formulas for propositional variables.

Its non- $\xi$ rule is the irreflexivity rule for $D_{n}$, viz

$\left(I R_{D_{n}}\right) \quad\left(p \wedge \neg D_{n} p\right) \rightarrow \phi / \phi, \quad$ provided that $p$ does not occur in $\phi$.

$B_{n}^{+}$is the extension of $A_{n}^{+}$with the axioms $M 1, M 2$ and $M 3$, and the Universal Generalization rule for every $\otimes_{i j}$ (i.e. $\left.\phi / \neg \otimes_{i j} \neg \phi\right)$.

Notions like derivation, theorems and such are defined as usual. Theoremhood of $\phi$ in $A_{n}^{+}$ $\left(B_{n}^{+}\right)$is denoted by $A_{n}^{+} \vdash \phi\left(B_{n}^{+} \vdash \phi\right)$. We will refer to $A_{n}^{+}$as the cylindric part of $B_{n}^{+}$.

Note that in Venema [13] we proved completeness of $A_{n}^{+}$with respect to cube validity. Now we can show the analogue for $B_{n}^{+}$with respect to mirror cubes:

Theorem 3.5 (Soundness \& Completeness) For any $C M M L_{n}$-formula $\phi$,

$$
B_{n}^{+} \vdash \phi \Longleftrightarrow \mathrm{MC}_{n} \models \phi
$$

\section{Proof.}

Soundness is left to the reader; completeness is essentially a straightforward corollary of Theorem 8.2 of Venema [14], Theorem 2.11 in Venema [13] and Proposition 3.3, but we have to work through some technical and rather cumbersome details.

Our strategy to prove completeness is as follows: first we extend the language $C M M L_{n}$ with the difference operator as a primitive symbol $D$, and we extend $B_{n}^{+}$to a derivation system $E B_{n}^{+}$in the extended language. Then we apply one of the mentioned theorems 
directly, obtaining a completeness result for $E B_{n}^{+}$with respect to a certain class $\mathrm{K}$ of frames. The third step of the proof is to show that $\mathrm{K}$ consists precisely of the disjoint unions of mirror cubes, whence $E B_{n}^{+}$is complete with respect to MC. Finally, we show that $E B_{n}^{+}$is conservative over $B_{n}^{+}$.

i So let us start with considering the language $X$, which is an extension of $C M M L_{n}$ with a new unary operator $D$. We abbreviate $\underline{D} \phi=\neg D \neg \phi$. The derivation system $E B_{n}^{+}$is obtained by adding the following axioms and rules to $B_{n}^{+}$:

$(D 1) \quad p \rightarrow \underline{D} D p$

$(D 2) \quad D D p \rightarrow(p \vee D p)$

$(D 3) \quad \diamond_{i} p \rightarrow(p \vee D p)$

$$
\otimes_{i j} p \rightarrow(p \vee D p)
$$

$\left(U G_{D}\right) \phi / \underline{D} \phi$

$\left(I R_{D}\right) \quad(p \wedge \neg D p) \rightarrow \phi / \phi$, provided that $p$ does not occur in $\phi$.

ii Note that all axioms of this system are in Sahlqvist tense form (cf. [14]), that all diamonds are self-conjugate, and that $D$ has indeed all the axioms and the rule $I R_{D}$ needed to make it the difference operator. Therefore, Theorem 8.2 of VENEma [14] yields that $E B_{n}^{+}$is sound and complete with respect to the class $\mathrm{K}_{n}$ of frames $\mathfrak{F}$ such that (1) all axioms of $E B_{n}^{+}$are valid in $\mathfrak{F}$ and (2) the accessibility relation of $R_{D_{n}}$ is irreflexive.

iii We will now prove that $\mathrm{K}_{n}$ is precisely the class of disjoint unions of mirror cubes, or formally

$$
\mathrm{K}_{n}=\mathbf{P}_{\mathbf{f}}\left(\mathrm{MC}_{n}\right)
$$

The inclusion $\supseteq$ is trivial, so we concentrate on the other direction. Let $\mathfrak{F}$ be a frame in $\mathrm{K}_{n}$; consider the cylindric reduct of $\mathfrak{F}$, which must be a disjoint union of cubes by Theorem 2.11 of [13]. As $\mathfrak{F}$ is in $\mathrm{K}_{n}$, the $M$-axioms are valid in $\mathfrak{F}$, so it is a trivial consequence of Proposition 3.3 that $\mathfrak{F}$ is a disjoint union of mirror cubes.

iv Finally, to show that $E B_{n}^{+}$is conservative over $B_{n}^{+}$, we first define an embedding translation $(\cdot)^{-}$:

$$
\begin{array}{ll}
p^{-} & =p, \\
(D \phi)^{-} & =D_{n} \phi^{-}, \\
(\phi \wedge \psi)^{-} & =\phi^{-} \wedge \psi^{-}, \\
(\varnothing \phi)^{-} & =\varnothing \phi^{-} \quad \text { for } \varnothing \in\left\{\neg, \otimes_{i j}, \diamond_{i} \mid i, j<n\right\} .
\end{array}
$$

The essential claim is that for all $E B_{n}^{+}$-formulas $\phi$ we have

$$
E B_{n}^{+} \vdash \phi \Longleftrightarrow B_{n}^{+} \vdash \phi^{-} .
$$

The direction from right to left is trivial; the other direction is proved by induction on derivations in $E B_{n}^{+}$.

For the basic step, let $\phi$ be an axiom of $E B_{n}^{+}$. Unless $\phi$ is of the form $\otimes_{i j} p \rightarrow(p \vee D p)$, the fact that $B_{n}^{+} \vdash \phi^{-}$follows from the proof of Theorem 3.4 in VEnEma [13]. For the remaining case, we have $B_{n}^{+} \vdash \phi$ by the following derivation:

$$
\begin{aligned}
& \otimes_{i j} p \rightarrow \diamond_{i}\left(\delta_{i j} \wedge \diamond_{j} p\right) \quad \text { (axiom) } \\
& \otimes_{i j} p \rightarrow \diamond_{i}\left(\delta_{i j} \wedge\left(p \vee D_{n} p\right)\right) \quad\left(\text { as } A_{n}^{+} \vdash\left[\diamond_{j} p \rightarrow(p \vee D p)\right]^{-}\right) \\
& \otimes_{i j} p \rightarrow \diamond_{i}\left(p \vee D_{n} p\right) \quad \text { (monotonicity of } \diamond_{i} \text { ) } \\
& \otimes_{i j} p \rightarrow\left[\left(p \vee D_{n} p\right) \vee D_{n}\left(p \vee D_{n} p\right)\right] \quad\left(\text { as } A_{n}^{+} \vdash\left[\diamond_{i} \psi \rightarrow(\psi \vee D \psi)\right]^{-}\right) \\
& \otimes_{i j} p \rightarrow\left(p \vee D_{n} p \vee D_{n} p \vee D_{n} D_{n} p\right) \quad \text { (distributivity of } D_{n} \text { ) } \\
& \otimes_{i j} p \rightarrow\left(p \vee D_{n} p\right) \quad\left(\text { as } A_{n}^{+} \vdash[D D p \rightarrow(p \vee D p)]^{-}\right)
\end{aligned}
$$


All cases of the induction step of the proof are either trivial (Universal Generalization for $\otimes_{i j}$ ), or can be found again in Venema [13]. This proves (3); the fact that $E B_{n}^{+}$is conservative over $B_{n}^{+}$then follows by the observation that for a $C M M L_{n}$-formula $\phi$ we have $\phi^{-}=\phi$.

\section{Representable Polyadic Equality Algebras}

In this section we look at the algebraic connection of first-order logic with finitely many variables, and of our modal system $C M M L$. In the algebraic approach towards first-order model theory, one is interested in such operations on the power set algebra of models as are defined by the semantic truth definition of the operators of the language.

Consider the case of $L_{n}$ : let $\phi(i / j)$ denote the $L_{n}$-formula $\phi$ where the variables $v_{i}$ and $v_{j}$ are interchanged, and let for an arbitrary structure $\mathfrak{M}, \phi^{\mathfrak{M}}$ denote the set of tuples where $\phi$ holds. Then

$$
\begin{aligned}
& \left(\exists v_{i} \phi\right)^{\mathfrak{M}}=\left\{u \in \in^{n} U \mid \exists t\left[u \equiv_{i} t \& t \in \phi^{\mathfrak{M}}\right]\right\} \\
& \phi(i / j)^{\mathfrak{M}}=\left\{u \in{ }^{n} U \mid \exists t\left[u \bowtie_{i j} t \& t \in \phi^{\mathfrak{M}}\right]\right\} \\
& (\neg \phi)^{\mathfrak{M}}={ }^{n} U-\phi^{\mathfrak{M}} \\
& \text { etc. }
\end{aligned}
$$

So algebraists study (besides the booleans) the operations $C_{i}, S_{i j}$ and $D_{i j}$ on the power set algebra of ${ }^{n} U$, given by:

$$
\begin{aligned}
& C_{i}(X)=\left\{u \in^{n} U \mid \exists t\left[u \equiv_{i} t \& t \in X\right]\right\}, \\
& S_{i j}(X)=\left\{u \in^{n} U \mid \exists t\left[u \bowtie_{i j} t \& v \in X\right]\right\}, \\
& D_{i j}(X)=\left\{u \in^{n} U \mid u_{i}=u_{j}\right\} .
\end{aligned}
$$

In other words, one is interested in the complex algebras of structures, and just like in the modal approach, one abstracts away from this intended class of algebras where the elements are 'real' relations to the class of all boolean algebras with operators of the appropriate similarity type. An aim of the algebraic theory is then to find necessary and sufficient criteria for the representability of an abstract algebra as a concrete algebra of relations.

Definition 4.1 A PEA-type algebra of dimension $n$ is an algebra of the form

$$
\mathfrak{A}=\left(A,+,-, c_{i}, d_{i j}, s_{i j}\right)_{i, j<n}
$$

where $(A,+,-)$ is a boolean algebra, every $d_{i j}$ a constant and every $c_{i}$ and every $s_{i j}$ a normal, additive, unary operator.

We define the following classes of algebras: $\mathrm{FCMS}_{n}$ is the class of full n-dimensional polyadic equality algebras over some set $U$, i.e. algebras of the form

$$
\left(\mathcal{P}\left({ }^{n} U\right), \cup,-, C_{i}, D_{i j}, S_{i j}\right)_{i, j<n},
$$

with $C_{i}, D_{i j}$ and $S_{i j}$ as defined in (4). The class RPEA of $_{n}$-dimensional representable polyadic equality algebras consists of all 'real' relational algebras, i.e. of the subalgebras of direct products of algebras in $\mathrm{FCMS}_{n}$.

For the similarity type without the $s_{i j}$-operators, we have analogous terminology: CA-type algebras, full cylindric set algebras (FCS) and representable cylindric algebras (RCA). 
Polyadic Equality Algebras (or Polyadic Algebras with Equality, as they were called in the past) are known from the literature on algebraic logic ${ }^{5}$, mainly through the work of Halmos, cf. Halmos [5] for his collected articles on these algebras and on their diagonal free reducts, the Polyadic Algebras. More recent surveys on algebras of relations can be found in HENKIN, Monk \& TARski [6] or NÉmeti [10].

The symmetry with the classes of relational frames defined in the previous section is obvious, for instance the class $\mathrm{FCMS}_{n}$ consists of the complex algebras of the mirror cubes. Therefore, recursively enumerating the 'true' polyadic equality equations (i.e. the equational theory of $\mathrm{FCMS}_{n}$ ) and axiomatizing the modal theory of $\mathrm{MC}_{n}$ amounts to the same thing. Recall from the previous section that we used the 'defined difference operator' $D_{n}$ in our axiomatization; in the algebraic language we define

$$
d_{n} y=\bigvee_{j \neq i} c_{j}\left(d_{i j} \cdot c_{i}\left(-d_{i j} \cdot c_{0} \ldots c_{i-1} c_{i+1} \ldots c_{n-1} x\right)\right)
$$

Definition 4.2 Let $n$ be an arbitrary natural number. Let $\Omega_{n}$ be the smallest set of PEA-type equations ${ }^{6}$ satisfying

1. $\Omega_{n}$ contains axioms stating that the algebra is a Boolean Algebra with (normal and additive) Operators,

2. $\Omega_{n}$ contains the following axioms:
$\left(C 1_{i}\right) \quad c_{i} 0=0$
$\left(C 2_{i}\right) \quad x \leq c_{i} x$
$\left(C 3_{i}\right) \quad c_{i}\left(x \cdot c_{i} y\right) \leq c_{i} x \cdot c_{i} y$
$\left(C 4_{i j} \quad c_{i} c_{j} x \leq c_{j} c_{i} x\right.$
$\left(C 5_{i}\right) \quad d_{i i}=1$
$\left.\left(C 6_{i j}\right) \quad c_{i}\left(d_{i j} \cdot x\right) \cdot c_{i}\left(d_{i j} \cdot-x\right)\right)=0$
$\left(C 7_{i j k}\right) \quad d_{i j}=c_{k}\left(d_{i k} \cdot d_{k j}\right)$
$\left(C 8_{i j}\right) \quad d_{i j} \cdot c_{i}\left(-x \cdot c_{j} x\right) \leq c_{j}\left(-d_{i j} \cdot c_{i} x\right)$
$\left(P 1_{i j}\right) \quad p_{i j} x=-p_{i j}-x$
$\left(P 2_{i j}\right) \quad x \leq-p_{i j}-p_{i j} x$
$\left(P 3_{i j}\right) \quad p_{i j} x \leq c_{i}\left(d_{i j} \cdot c_{j} x\right) \cdot c_{j}\left(d_{i} j \cdot c_{i} x\right)$

3. $\Omega_{n}$ is closed under the ordinary algebraic deduction rules (i.e. identity, symmetry, transitivity, substitution and replacement),

4. $\Omega_{n}$ is closed under the algebraic version of the $D_{n}$-irreflexivity rule:

$$
\begin{aligned}
& y \cdot-d_{n}(y) \leq t\left(x_{0}, \ldots, x_{n-1}\right) / t\left(x_{0}, \ldots, x_{n-1}\right)=1 \\
& \text { if } y \text { does not occur among the } \vec{x} .
\end{aligned}
$$

$\Omega_{n}$ is easily seen to be an algebraic counterpart of $B_{n}^{+}$(in a sense to be made precise in (6) below). So the following result is an almost immediate corollary of Theorem 3.5.

\footnotetext{
${ }^{5}$ Note that our approach slightly deviates from the standard definition, where operations $s_{\sigma}$ are considered for every map $\sigma: n \mapsto n$; however, the fact that we only treat the finite-dimensional case ensures that every such operator is term-definable in our system, cf. the discussion in section 2 .

${ }^{6}$ We will use $s \leq t$ to abbreviate the equation $s \cdot t=s$.
} 


\section{Theorem 4.3 (Enumerating 'true' Polyadic Equality Equations) For every $n$ :}

$$
\Omega_{n}=\operatorname{Equ}\left(\mathrm{RPEA}_{n}\right)
$$

\section{Proof.}

Let $\mu$ be the translation mapping $P E A$-terms to their corresponding modal formulas (i.e $\mu$ is inductively defined with clauses like $\mu\left(s_{i j} t\right)=\otimes_{i j} \mu t$, etc.) By a standard inductive proof on the length of derivations one proves that for all equations $t_{0}=t_{1}$ one has

$$
t_{0}=t_{1} \in \Omega_{n} \Longleftrightarrow B_{n}^{+} \vdash \mu t_{0} \leftrightarrow \mu t_{1} .
$$

The observation that $\mathrm{FCMS}_{n}$ is the class of complex algebras of frames in $\mathrm{MC}_{n}$ implies that for all equations $t_{0}=t_{1}$ :

$$
\mathrm{FCMS}_{n} \models t_{0}=t_{1} \Longleftrightarrow \mathrm{MC}_{n} \models \mu t_{0} \leftrightarrow \mu t_{1} .
$$

By these two observations, taken together with Theorem 3.5, we find that

$$
\Omega_{n}=\operatorname{Equ}\left(\mathrm{FCMS}_{n}\right)
$$

which is sufficient to prove the theorem, as RPEA ${ }_{n}$ is the variety generated by $\mathrm{FCMS}_{n}$, and therefore shares its equational theory.

Note that in VenEma [13] it was proved that the system $\Sigma_{n}$ obtained by leaving out from $\Omega_{n}$ all axioms referring to the substitution operators, is a recursive enumeration of $\operatorname{Equ}\left(\mathrm{RCA}_{n}\right)$. So in contrast to the results obtained by Andréka and Tuza mentioned before, $\Omega_{n}$ is an extension of $\Sigma_{n}$ with finitely many axioms. In other words, for non-finite axiomatizability results, the kind of the derivation system one is referring to, plays an essential rôle.

The algebraically oriented reader might find (5) not so appealing - it is the literal translation of the modal rule $\left(I R_{D_{n}}\right)$. A perhaps more intuitive variant can be found as follows: define $\mho_{n}$ as the union of the equational axioms of $\Omega_{n}$ plus the non-equational axiom

$$
\forall x \exists y\left(x \neq 0 \rightarrow\left(0 \neq y \cdot-d_{n} y \wedge y \cdot-d_{n} y \leq x\right)\right) .
$$

To explain the meaning of (7), let us note that it is valid in $\mathrm{FCMS}_{n}$. The crucial fact is that in a full $n$-dimensional polyadic equality algebra $\mathfrak{A}=\left(\mathcal{P}\left({ }^{n} U\right), \cup,-, C_{i}, D_{i j}, S_{i j}\right)_{i, j<n}$ we have, for an arbitrary subset $Y$ of ${ }^{n} U$ :

$$
d_{n}(Y)= \begin{cases}\varnothing & \text { if } Y=\varnothing \\ { }^{n} U \backslash Y & \text { if } Y \text { is a singleton, } \\ { }^{n} U & \text { otherwise. }\end{cases}
$$

We leave the proof of this claim to the reader; the key observation is that over the class of mirror cubes, $D_{n}$ (the modal version of $d_{n}$ ) has the inequality relation as its accessibility relation. Now it is straightforward to show the validity of (7) in $\mathrm{FCMS}_{n}$ : let $U$ be some set, and $X$ a non-empty subset of ${ }^{n} U$. Then $X$ contains an element $x$. Clearly we find

$$
\varnothing \neq\{x\}=\{x\} \cap-d_{n}(\{x\}) \subseteq X,
$$

which is what we were after. 
Note that (7) can be replaced by a $\forall \exists$-formula with an equational matrix. For, let $c^{n}$ be the term defined by

$$
c^{n} x=c_{0} \ldots c_{n-1} x
$$

then, given the axioms $(C 1) \ldots(C 4), c^{n}$ has the property that over simple algebras

$$
c^{n} x= \begin{cases}0 & \text { if } x=0 \\ 1 & \text { if } x \neq 0,\end{cases}
$$

(we are in a discriminator variety!) whence (7) can be replaced by

$$
\forall x \exists y\left[c^{n} x \leq c^{n}\left(y \cdot-d_{n} y\right) \cdot-c^{n}\left(y \cdot-d_{n} y \cdot-x\right)\right] .
$$

Now let us give a brief proof of the equivalence of $\Omega_{n}$ and $\mho_{n}$, i.e. prove that for any equation $\eta$ :

$$
\eta \in \Omega_{n} \Longleftrightarrow \mho_{n} \vdash \eta
$$

The direction from right to left is almost immediate: if $\mho_{n} \vdash \eta$, then $\mathrm{FCMS}_{n} \models \eta$ by the soundness of $\mho_{n}$, and so $\eta \in \Omega_{n}$ by the completeness of $\Omega_{n}$. The other direction is proved by induction on the length of $\Omega_{n}$-derivations. The only important step is where the unorthodox derivation rule (5) is involved: we have to show that it can be derived form $\mho_{n}$. So, suppose that

$$
\mho_{n} \vdash y \cdot-d_{n} y \leq t(\vec{x}),
$$

where $y$ does not occur among the $t(\vec{x})$. As an instantiation of $(7)$, we have

$$
\mho_{n} \vdash \exists y\left[-t(\vec{x}) \neq 0 \rightarrow\left[0 \neq y \cdot-d_{n} y \wedge y \cdot-d_{n} y \leq-t(\vec{x})\right]\right]
$$

So

$$
\mho_{n} \vdash t(\vec{x}) \neq 1 \rightarrow \exists y\left[0 \neq y \cdot-d_{n} y \wedge y \cdot-d_{n} y \leq-t(\vec{x}) \wedge y \cdot-d_{n} y \leq t(\vec{x})\right],
$$

whence

$$
\mho_{n} \vdash t(\vec{x}) \neq 1 \rightarrow \exists y\left[0 \neq y \cdot-d_{n} y \wedge y \cdot-d_{n} y \leq 0\right] .
$$

From this it follows that $\mho_{n} \vdash t(\vec{x})=1$; so (5) is a derived rule of $\mho_{n}$. This proves (8).

So, what is really behind the fact that a simple finite equational extension of the nonequational axiomatization of $\mathrm{RCA}_{n}$ gives an axiomatization for $\mathrm{RPEA}_{n}$, is that the universalexistential axiom takes us down to the class $\mathbf{P}\left(\mathrm{FCS}_{n}\right)$ of direct products of complex algebras of cubes, and that on this class, the class $\mathbf{P}\left(\mathrm{FCMS}_{n}\right)$ of direct products of complex algebras of mirror cubes is definable by finitely many equations.

Finally, it is interesting to note that recently, generalizations to the results reported on here and in Venema [13] were obtained by Sz. Mikulás (cf. [8]). Applying an algebraic representation theorem, Mikulás finds finite complete derivation systems for various logics and classes of representable algebras of relations. A nice aspect of his approach is that his unorthodox rules do not refer to the algebraic/modal difference operator: for instance, one of his completeness results concerns $n$-variable logic without equality — a system in which the $D$-operator is not even definable. 


\section{References}

[1] H. Andréka, Complexity of Equations Valid in Algebras of Relations, Third Doctoral Dissertation, Budapest, 1991.

[2] H. Andréka \& I. Németi, Lecture given at "Logic at Work"; Applied Logic Conference, Amsterdam, 1992.

[3] H. Andréka \& Zs. Tuza, "Nonfinite axiomatizability of the polyadic operations in algebraic logic", Abstracts of the American Mathematical Society 9 (1988), 500, *88T-03-264.

[4] D.M. Gabbay, "An irreflexivity lemma with applications to axiomatizations of conditions on linear frames", in: [9], pp. 67-89

[5] P.R. Halmos, Algebraic Logic, Chelsea Publishing Company, New York, 1962.

[6] L. Henkin, J. D. Monk \& A. Tarski, Cylindric Algebras, North-Holland, Amsterdam, part I, 1971, part II, 1985.

[7] S. Kanger (ed.), Proc. of the Third Scandinavian Logic Symposium Uppsala 1973, NorthHolland, Amsterdam, 1975.

[8] Sz. Mikulás, Gabbay-style calculi, manuscript, Mathematical Institute of the Hungarian Academy of Sciences, Budapest, 1993.

[9] U. Mönnich (ed.), Aspects of Philosophical Logic, Reidel, Dordrecht, 1981.

[10] I. Németi, "Algebraizations of quantifier logics: an introductory overview", Studia Logica 50 (1991) 485-570.

[11] M. de Rijke, "The modal logic of inequality", Journal of Symbolic Logic, 57 (1992) $566-584$.

[12] H. Sahlqvist, "Completeness and correspondence in the first and second order semantics for modal logic", in: [7], pp. 110-143.

[13] Y. Venema, "Cylindric Modal Logic", Logic Group Preprint Series 105, Department of Philosophy, Utrecht University, 1993, to appear in Journal of Symbolic Logic.

[14] Y. Venema, "Derivation rules as anti-axioms", Journal of Symbolic Logic, 58 (1993) 1003-1034. 